\title{
PROSPETTIVE E LIMITI DELL'ECONOMIA QUANTITATIVA
}

\author{
LUIGI PASINETTI $(*)$
}

RiasSunTO. -L'economia politica è, tra le scienze umane, quella che maggiormente sperimenta l'attrazione verso i metodi quantitativi e in particolare verso la matematica e la statistica. Recentemente questa attrazione si è fatta ancor più forte che in passato, al punto che non è raro trovare, tra gli economisti, dei teorici che amerebbero vedere l'economia considerata come un ramo della matematica applicata. Ma sarebbe davvero utile convogliare l'economia tra le scienze naturali o addirittura spingerla al ruolo di un ramo della matematica applicata?

Una attenta riflessione porta ben presto alla constatazione che il rapporto tra economia e matematica è molto più complesso di quanto possa sembrare a prima vista.

L'autore sottolinea che ci sono almeno quattro caratteristiche che differenziano l'economia politica dalle scienze naturali: l'oggetto di studio; la finalità della ricerca economica tesa ad influenzare l'oggetto di studio; la sua caratteristica di disciplina per certi aspetti positiva e per altri aspetti normativa; il coinvolgimento dei giudizi di valore.

Per illustrare queste differenze e inquadrare la dimensione autonoma dell'economia politica, l'autore ricorre ad una rassegna critica delle opinioni di alcuni economisti di rilievo, che hanno dedicato contributi all'argomento qui in discussione. Specifici riferimenti vengono fatti a Marshall, Keynes, Hicks, Morishima e - tra gli economisti maggiormente presenti nelle nostre discussioni - a Sraffa e Sen, ai quali ultimi l'autore si sente più vicino.

ABSTRACT. - Among the human sciences, economics is the one experimenting a high degree of attraction towards quantitative methods and, in particular, towards mathematics and statistics. In our times, this attraction is even stronger than in the past, so that it is customary to find, among economists, theoreticians aiming at presenting economics as a branch of applied mathematics. Is it really useful to include economics among the natural sciences, or even to consider it as a branch of applied mathematics?

(*) Istituto Lombardo Accademia di Scienze e Lettere, Università Cattolica del Sacro Cuore di Milano. 
A careful reflection leads quickly to the awareness that the relations between economics and mathematics are much more complex than one can see at first sight.

The Author underlines that there are at least four characteristics which differentiate political economy from natural sciences: the object of study; the purpose of economic research (which has an influence on the object of study); its characteristic of being a discipline which is both positive and normative; the dependence on value judgements.

To illustrate such differences, and to focus on the autonomous dimension of political economy, the Author critically reviews the opinions of some famous economists, who contributed to the subject under discussion. Specific references are made to Marshall, Keynes, Hicks, Morishima and - among the economists more relevant to the present discussion - Sraffa and Sen, to whom the Author feels closer than to the others.

\section{PREMESSA}

Questo incontro di studio, dedicato alle applicazioni dello strumento matematico alle elaborazioni economiche non poteva arrivare in un momento più critico di quello che si presenta attualmente nelle nostre università per l'insegnamento dell'economia politica. Dobbiamo essere grati all'Istituto Lombardo, Accademia di Scienze e Lettere, e ai professori Nicola e Zanella, ispiratori di questo Convegno, per avercelo proposto.

Il momento è critico perché, tra le scienze umane, l'economia politica è quella che sperimenta in modo più forte l'attrazione dei metodi quantitativi e quindi degli strumenti analitici che sono stati sviluppati per le scienze naturali. Ciò non è del tutto sorprendente, e ancor meno disdicevole. Da tempo, gli economisti mostrano una forte ammirazione per l'ordine, la precisione - e i successi - delle scienze naturali. L'attrazione è quindi giustificata. Recentemente si è fatta ancora più forte che nel passato. In questo contesto, una formazione prevalentemente tecnicistica (dove le tecniche da usare sono la matematica, l'econometria, le nuove tecniche statistiche, come l'analisi fattoriale, con una presenza ridotta della statistica descrittiva) ha influito su un processo di emarginazione e perfino di eliminazione, dai piani di studio delle Facoltà di Economia, della storia economica, della storia del pensiero economico e dello studio delle istituzioni, un tempo considerate componenti essenziali di una cultura economica completa. La conseguenza è talvolta una tendenza a percepire l'economista come un tecnico, più che come uno scienziato sociale.

Non può sfuggire che l'economia politica sembra stia diventando sempre più una scienza di calcolo, frazionata in iper-specialismi, poco o 
nulla propensa a dialogare con le altre scienze umane; talvolta persino restia a definirsi tale. In sede accademica c'è addirittura chi si chiede se non sia auspicabile una fuoriuscita dell'economia politica dall'ambito delle scienze umane. (Queste osservazioni reiterano quelle già fatte in Pasinetti e Roncaglia, 2006).

Ma davvero sarebbe auspicabile sospingere l'economia politica al ruolo di un ramo della matematica applicata? E all'uso di metodologie e criteri di valutazione propri delle scienze naturali?

\section{L’ECONOMIA COME SCIENZA UMANA - LA RICCHEZZA DELL'ETEROGENEITÀ}

Per rispondere a questi quesiti colgo volentieri questa occasione per riprendere brevemente e fondere alcuni contributi presentati in precedenza sull'argomento. Innanzitutto, in un Convegno della European Science Foundation, indetto per discutere sul significato di progresso nelle varie scienze naturali e sociali, cercai di enucleare almeno quattro caratteristiche che differenziano l'economia politica dalle scienze naturali (Pasinetti, 1985, pp. 183-186).

Prima di tutto, l'oggetto di studio. A differenza per esempio della fisica o dell'astronomia o di altre scienze naturali, l'oggetto degli studi economici cambia continuamente. Quando, più di due millenni or sono, Tolomeo osservava nel cielo i pianeti e le stelle, guardava esattamente lo stesso universo che noi oggi esploriamo con l'aiuto dei grandi telescopi: si pensi al telescopio spaziale Hubble e ai radiotelescopi. L'universo osservabile non è cambiato (o è cambiato in modo a noi quasi impercettibile). Possiamo semplicemente indagarlo in modo migliore; siamo in grado di penetrarlo maggiormente e più in profondità. Non è questo il caso dell'economia. Quando Adam Smith, soltanto poco più di due secoli fa, indagava la società britannica che stava emergendo dalla Rivoluzione Industriale, osservava qualcosa che era non già in modo impercettibile ma profondamente diverso, rispetto, diciamo, all'Eurolandia di oggi.

In secondo luogo, la ricerca economica (ancora una volta a differenza della fisica) tenderà il più delle volte ad essere condotta con il proposito di influenzare gli eventi che sono oggetto di studio. L'economista non può semplicemente stare in disparte ad osservare, o spiegare in modo distaccato. Per esempio, quando cerchiamo di capire 
le cause della disoccupazione o dell'inflazione, lo facciamo ovviamente con l'obiettivo di escogitare misure o provvedimenti per contrastare e superare questi fenomeni indesiderati.

In terzo luogo, l'economia è per certi aspetti teoria positiva, ma per altri aspetti essa copre componenti esplicitamente normative. Tende ad indicarci, o a cercare di definire, come i fenomeni studiati dovrebbero essere, e quindi a spronare lo stesso agire umano. Per questo Keynes la definiva "con forza una scienza morale". Non così la fisica o l'astronomia, che possono interessarsi di galassie e persino di ipotetici altri universi, senza poterne minimamente cambiare o deviare le caratteristiche o traiettorie. Scienziati con opinioni e convincimenti anche molto lontani, o addirittura opposti, non troveranno difficoltà a concludere che "la mela di Newton", per citare un aneddoto famoso e largamente riferito, se rimessa al suo posto, ricade proprio con la stessa accelerazione di gravità.

In quarto luogo, molti pronunciamenti su questioni economiche non possono evitare giudizi di valore. Si pensi ai provvedimenti che influenzano la distribuzione del reddito e della ricchezza, o che riguardano il sistema fiscale, il livello dei prezzi, i tassi d'interesse e così via. Per di più, e in modo significativo nel nostro caso, giudizi di valore possono già essere individuati alla base della "visione del mondo" che inevitabilmente giace dietro l'astratto teorizzare economico.

Val la pena di richiamare come Keynes abbia scritto a più riprese su questo argomento, in modo esteso e molto interessante. Vi si è riferito fra l'altro nel suo saggio biografico dedicato al suo maestro, Alfred Marshall, mostrando come questi, nei suoi studi precedenti - di filosofia, di psicologia, di teologia, di metafisica - si fosse inizialmente proposto di dedicarsi alla fisica molecolare, poi alla matematica, per poi spostarsi verso "i fondamenti filosofici della conoscenza", scegliendo alla fine l'economia politica tra le "scienze morali", e tuttavia prendendo vantaggio notevole della geometria e dell'algebra, ma astenendosi sempre dall'usarle esplicitamente (Keynes 1933 [1972], pp. 162-166).

Lo stesso Keynes, in una lettera al discepolo e amico Roy Harrod, si soffermò a lungo a descrivere quanto sostanziale sia la differenza tra l'oggetto di studio dell'economia e quello della fisica. Riferendosi al succitato aneddoto della caduta della mela dall'albero, Keynes scrive:

"[Sarebbe] come se dipendesse dai motivi della mela che cade dall'albero se valga la pena cadere sul terreno, e se il terreno desidera che la mela cada, 
e dall'errore di calcolo che da parte della mela si compie nel valutare quanto essa dista dal centro della terra" (Keynes 1938 [1973], p.300).

\section{E aggiungeva:}

"Nella chimica e nella fisica come in altre scienze, l'obiettivo dell'esperimento è di trovare le misure effettive delle varie quantità e i fattori che appaiono, in un'equazione o in una formula. Il lavoro, quando è stato svolto, viene svolto una volta per tutte. In economia questo non accade, e talvolta il convertire un modello in una formula quantitativa significa distruggere la sua utilità come strumento di pensiero" (ibidem).

Ecco perché Keynes pensava e voleva "enfatizzare con forza” l'osservazione che "l'economia è una scienza morale".

Scriveva sempre Keynes che le qualità necessarie per fare un buon economista sono molteplici, e i "buoni economisti sono scarsi perché il dono nell'utilizzare la 'vigilante osservazione' sui fatti per scegliere dei buoni modelli economici, sebbene sia qualità che non richiede un'elevata specializzazione tecnica, appare comunque una qualità intellettuale molto rara" (Ibidem).

Si tratta, come si può percepire, di differenze per nulla marginali, che richiedono ulteriori riflessioni proprio sul terreno di come procede la ricerca scientifica. Se la materia indagata dall'economia è così diversa da quella indagata dalla fisica, è ragionevole pensare che i progressi in economia non possano avvenire con le stesse regole in uso nella fisica. Thomas Kuhn, il noto studioso di storia delle scienze fisiche, forgiò, in un famoso libro (Kuhn 1962), il termine di "paradigma scientifico". Egli osservava che le scienze naturali sono caratterizzate da fasi di ordinata e lenta accumulazione di conoscenze (le fasi di "scienza normale", come egli le definì), e da fasi (più rare) di grandi cambiamenti, di radicali rotture con il passato ("le rivoluzioni scientifiche"). In entrambe le fasi vi sono miglioramenti, ma nel lungo periodo sono le "rivoluzioni scientifiche”, secondo Kuhn, che fanno progredire la scienza, perché è in questa fase che un vecchio paradigma scientifico viene scalzato e sostituito da un nuovo paradigma, di capacità esplicativa maggiore. E' attraverso questa sorta di evoluzione darwiniana, da un paradigma all'altro, che la scienza naturale sperimenta il progresso.

La domanda che ci si può porre è ora se l'economia politica si evolva allo stesso modo. La risposta sembrerebbe negativa. Risulta infatti che in economia (e nelle scienze sociali in genere) esista una mol- 
teplicità di teorie e di approcci molto più variegata che nelle scienze naturali. I grandi paradigmi della teoria economica, più che rimpiazzarsi, sembrano coesistere nel tempo, anche se con diversa intensità, con vicende alterne. Proprio perché la realtà di un sistema economico è il continuo movimento, non sorprende che più teorie cerchino di coglierne ed interpretarne, attraverso la ricerca, aspetti diversi, certe volte addirittura contrastanti, anche se tutti in qualche modo rilevanti.

John Hicks, premio Nobel per l'economia nel 1971, osservava, scrivendo proprio sulle "rivoluzioni scientifiche" in economia (vedi Hicks 1976), come le teorie in questa disciplina possono essere altrettanto penetranti che nelle scienze naturali, ma enormemente più finalizzate. Per questo Hicks assimila le teorie economiche a dei "paraocchi”. Permettono di focalizzare l'attenzione (e l'analisi) su un tema, lasciando in ombra il resto. Il punto cruciale, però, che va sottolineato, è che una realtà che cambia può trasformare un paraocchi illuminante in un paraocchi irrilevante o addirittura fuorviante. Questo ci porta a comprendere meglio perché, in questa disciplina come in molte altre scienze sociali, non si assista ad un progresso lineare, cumulativo e irreversibile. Prevale la varietà di vedute. Più che ad un susseguirsi di paradigmi, si assiste alla coesistenza di una pluralità di paradigmi, che procedono da "visioni del mondo" diverse. Così, di fatto, almeno fino ad oggi, l'eterogeneità delle teorie economiche ha prevalso sull'egemonia di una singola teoria, in ciò venendo a costituire un elemento di ricchezza intellettuale dell'economia politica, e non già una limitazione delle sue prospettive. La giustificazione è intuitiva: se il mondo dell'economia cambia così rapidamente, non è fruttuoso (e si capisce come possa diventare in certi casi fuorviante) osservare la realtà servendosi di un unico "paraocchi". Sembrerebbe meno rischioso, e in effetti molto più fecondo, tenere a disposizione più "paraocchi" alla bisogna (si veda anche Pasinetti e Roncaglia, 2006).

\section{MATEMATICA ED ECONOMIA: UN RAPPORTO PIÙ COMPLESSO DI QUANTO POSSA SEMBRARE A PRIMA VISTA}

Chi mi ha seguito fin qui, potrebbe ragionevolmente chiedersi: ma che nesso c'è tra l'adesione a un certo paradigma, nel senso di Kuhn, e l'applicazione della matematica in economia?

Non è la matematica, come tale, uno strumento logico che serve a 
render rigorose le elaborazioni cui viene applicata? Come tale, non è forse indifferente che essa venga usata all'interno di un particolare paradigma o di un altro? Queste domande sono pertinenti, ma le risposte non sono per nulla ovvie. Per illustrare quanto la questione sia in effetti complessa, nel caso delle scienze sociali e in modo particolare dell'economia politica, esemplificherò riferendomi a due noti economisti del nostro tempo: Piero Sraffa e Amartya Sen.

E qui mi torna utile riferirmi a un altro contributo (Pasinetti, 2004), che sembra particolarmente appropriato al tema che qui si sta discutendo. Piero Sraffa, come è noto, è autore di un volume straordinariamente conciso (Produzione di merci a mezzo di merci, 1960, abbr. PMmM): 100 pagine in tutto, che ha dato origine, per la sua radicale critica all'economia dominante, a una serie di discussioni che sembra interminabile. In questo volumetto, la matematica è usata non solo con parsimonia, ma con malcelata diffidenza. Eppure, nel sottofondo, la matematica non solo è presente, ma in certi punti sembra addirittura essenziale. In effetti è stato proprio grazie all'utilizzo dell'algebra delle matrici non-negative e dei relativi teoremi di Perron-Frobenius che è stato possibile, nel corso di alcuni anni, riuscire più facilmente a rendere comprensibili le innovazioni dell'analisi di Sraffa, soprattutto nelle esposizioni didattiche del suo modello (si vedano Newman, 1962; Dominedò, 1962; Pasinetti, 1975). Si è stati in grado di considerare tutta una serie di concetti chiave dell'analisi sraffiana, evitando la fatica che la originaria rappresentazione di PMmM comporta. E si è anche potuto andare oltre (vedi, per esempio, Pasinetti, 1973).

Proprio la traduzione dei concetti sraffiani in notazione matematica sembra aver giocato un ruolo decisivo nel promuovere l'interesse per PMmM e nella diffusione di tale schema teorico sulla scena internazionale. Se pensiamo, per esempio, alla famosa controversia sul capitale tra le due Cambridge (Inghilterra e USA) di fine anni '60, dobbiamo riconoscere che sarebbe stato impensabile una critica tanto serrata alla teoria economica dominante - e soprattutto sarebbe stato impensabile l'accettazione dei risultati di questa critica - senza l'utilizzo della notazione matematica, per il tramite della quale i contendenti si sono potuti esprimere e confrontare con un linguaggio comune. Inoltre, andando oltre il ruolo critico, l'utilizzo della notazione matematica ha consentito anche di estendere il modello di Sraffa ben oltre la sua originaria rappresentazione. Si pensi alla rappresentazione della parte più complessa dell'analisi sraffiana, quella che riguarda la produzione con- 
giunta, di cui il matematico Carlo Felice Manara (1968), membro effettivo di questa Accademia, ha dato una formulazione che è stata alla base di tutte le successive elaborazioni sraffiane riguardanti la produzione congiunta (vedi per esempio Schefold, 1971). Ma forse è proprio di questo che Sraffa diffidava. Perché?

Sraffa sapeva benissimo che la matematica, come tutti i linguaggi tecnici, non è totalmente neutrale rispetto all'oggetto che si propone di indagare. Non è ingiustificato il suo timore che l'uso della notazione matematica potesse introdurre distorsioni, enfatizzare certi aspetti, nasconderne altri.

$\mathrm{Ci}$ sono fin troppi casi nella storia del pensiero economico di quanto (e di come) il formalismo matematico abbia condizionato, e soggiogato alle sue esigenze, il ragionamento economico. Si pensi, per fare solo un esempio, al notevole ruolo che la matematica (nella fattispecie la necessità di usare funzioni convesse) ha avuto nel difendere fino al ridicolo la supposizione dei rendimenti decrescenti nella produzione economica. Non è affatto sorprendente che il modello dell'Equilibrio Economico Generale (EEG), cioè l'impianto analitico su cui si basa gran parte della teoria dominante, sia completamente assiomatizzato. Il fascino che questo modello esercita in termini matematici deriva dalla sua impeccabile coerenza interna, dall'esistenza di soluzioni di equilibrio e dalla sua parvenza di completezza (il sistema è chiuso!). Ma da un punto di vista strettamente economico esso manca totalmente l'obiettivo per cui una teoria dovrebbe essere costruita: la rilevanza empirica.

In effetti la generalità della teoria dell'EEG non sta tanto (anzi non sta affatto) nel cogliere aspetti importanti della realtà economica, sta invece nella sua capacità di cogliere un intero sistema di equazioni grazie ad assunti di partenza accettati esogenamente e grazie all'uso di ipotesi ad hoc (cioè di comodo) imposte al modello. Ma proprio questa necessaria rigidità delle premesse rende la teoria chiusa (dunque sorda) all'introduzione di ogni ipotesi alternativa. E' necessario fissare il tipo di agente economico (razionale), il tipo di preferenze e di tecnologia (date, interamente conosciute, e convesse), il tipo di attività svolta (scambio neutrale all'uso dello strumento monetario). E' persino necessario incorporare i beni che ancora non esistono (in pratica, assenza di futuro). La teoria emerge formalmente (vale a dire, matematicamente) impeccabile, ma rimane economicamente sterile (Pasinetti 2005).

L'impressione che si ha quindi, nel caso Sraffa, è che ai matematici egli cercasse di affidare il compito di controllo della coerenza interna 
della sua teoria e di esclusione di campi di soluzione solo formalmente possibili, ma privi di rilevanza economica. Per questo, egli è rimasto sempre scettico nell'utilizzare esplicitamente la notazione matematica per la costruzione e lo sviluppo dei suoi concetti teorici. Si potrebbe forse dire che Sraffa affidasse alla matematica un compito di euristica negativa, mentre abbia preferito di gran lunga fare assegnamento sul ragionamento logico per l'euristica positiva.

Non è facile dare una giustificazione totalmente plausibile di questa resistenza alla formalizzazione matematica.

Mi servirò tuttavia di un esempio emblematico che mi pare bene illustri questa ritrosia di Sraffa a lasciarsi condurre dalla logica matematica (o per meglio dire, dalla logica dei matematici). Questo esempio ci viene dalla "costruzione" della cosiddetta "merce tipo" di Sraffa. L'amico matematico Abram Besicovitch, dopo aver esaminato il sistema di equazioni che Sraffa gli aveva sottoposto, reagì sostenendo che di merci tipo ne potevano esistere molte o nessuna. Sraffa, seguendo la logica economica, sostenne con caparbia che la merce tipo esisteva, ed era unica. Alla fine, dopo una disputa che si protrasse per oltre un anno, fu proprio Besicovitch a convincersi e ad accettare il ragionamento di Sraffa. ${ }^{1}$ Il fatto è degno di nota non solo perchè dimostra la fondatezza (in questo caso sorprendente) della resistenza di Sraffa a lasciarsi condurre, nella sua costruzione teorica, da argomentazioni esclusivamente di tipo matematico, ma anche perché costituisce un monito per ciò che di norma succede; e cioè proprio l'opposto, come ben sappiamo. E' infatti la matematica di solito ad imporre la sua logica alla teorizzazione economica moderna.

Proprio con l'ottica di andare oltre la critica e di dar valore in termini positivi alla teoria di Sraffa, non solo è utile porsi il problema della giustificazione o meno dell'atteggiamento assunto da Sraffa in questo e in altre occasioni, ma è anche utile interrogarsi sulle possibilità a noi aperte per superare quella sua avversione.

Innanzitutto, è importante sottolineare che Sraffa, come premette all'inizio di PMmM, si muove nell'ambito della "teoria pura". Per "teoria pura" presumo che egli intendesse affermazioni sullo stato del mondo che fossero oggettive e incontrovertibili. Non ha mai nascosto

1 I particolari di questa disputa sono bene raccontati da De Vivo 2004: pp. $232-233$. 
la sua preoccupazione di evitare errori logici. L'ausilio che egli chiese alla matematica va senz'altro in questa direzione. Ma dobbiamo anche aggiungere che la matematica può essere usata (e, di fatto, è molto usata, anzi bisogna fare in modo che venga usata), non solo come strumento di controllo e verifica, ma anche come strumento d'analisi positiva capace di offrire nuovi sviluppi analitici.

Ma, per svolgere questo ruolo "costruttivo", la matematica richiede (anzi impone) regole ferree e restrittive. Per esempio, essa ha bisogno di muoversi su un terreno in cui sia chiara la distinzione tra incognite e dati, tra variabili e costanti; e in cui si possa fare (quando necessario) ipotesi ad boc tali da rendere maneggevoli la trasformazione e la semplificazione di sistemi che altrimenti rimarrebbero difficili da risolvere. E' proprio sulla scelta di queste ipotesi ad hoc che Sraffa non era disposto a cedere terreno.

Eppure è necessario andare oltre l'atteggiamento di Sraffa.

Senza intaccare il livello di astrazione, e senza compromettere in nulla la coerenza del ragionamento, non è difficile argomentare che esistono aspetti teorici verso i quali Sraffa si è astenuto dall'andare, ma che tuttavia appartengono proprio allo stadio della "teoria pura". Mi riferisco in particolare alla necessità di sviluppare il modello di PMmM in termini dinamici.

Sraffa pensava che la teoria pura, proprio per le ragioni di incontrovertibilità dei suoi assunti, potesse solo "fotografare" un sistema economico. E questo può spiegare la estrema cautela con cui procede in PMmM. Ma io penso che, senza perdere la sua natura, la "teoria pura" possa andare oltre la "fotografia"; possa cioè anche "filmare" un sistema economico in movimento. Sappiamo che il mondo, soprattutto quello industriale, è in continua evoluzione e la direzione di alcuni dei suoi movimenti contengono caratteristiche di persistenza che sono tipiche di un primo stadio di analisi economica (quello concernente le relazioni fondamentali). Si pensi ai progressivi e inevitabili cambiamenti della produttività, alla tendenza verso la meccanizzazione dei processi produttivi, alle tendenze implicite nella legge di Engels nel condizionare la domanda dei consumatori (vedi per esempio Pasinetti 1993); il tutto indipendentemente dagli assetti istituzionali e da ipotesi specifiche sul comportamento umano (si veda, su questo aspetto, anche Pasinetti, 2005).

E qui mi viene in aiuto il secondo economista che ho richiamato: Amartya Sen. 
Nel suo intervento al "Festival della matematica" tenutosi recentemente a Roma² (2008), Amartya Sen, premio Nobel per l'Economia, 1998, si è interrogato sulla rilevanza che il ragionamento matematico attualmente riveste nelle scienze sociali e particolarmente in quelle economiche, chiedendosi se queste ne risultino arricchite o impoverite. Egli ha senz'altro evidenziato il solido nesso storico che esiste tra l'evoluzione dell'economia e quella della matematica (e l'utile funzione di quest'ultima come strumento di indagine). Amartya Sen ha però anche sottolineato che "un uso fruttuoso del ragionamento matematico in economia richiede di valutare criticamente il tipo di matematica da usare per ogni problema", tenendo conto "della domanda dello scienziato sociale" e non solo "dell'offerta del matematico". La prospettiva di Sen si è estesa all'opportunità di fare ricorso a tecniche matematiche ancora non sviluppate: molte variabili economiche, sociali e politiche non possiedono, infatti, l'esattezza e la precisione tipica delle grandezze indagate dalle scienze naturali, ed è quindi possibile che i formalismi matematici attualmente a disposizione siano inadeguati a trattare alcune delle complessità sociali oggetto di indagine. In un simile contesto, il rischio che la scienza economica corre è quello di risultare addirittura limitata dall'incapacità delle strutture matematiche esistenti a cogliere variabili e relazioni che pure sono fondamentali nel contesto socioeconomico. E' possibile - ha continuato Sen - che i formalismi matematici attualmente a disposizione, anche presi nel loro insieme, siano inadeguati per trattare alcune delle complessità sociali di cui la scienza economica deve occuparsi. Certi economisti si sono ostinati a utilizzare solo tecniche matematiche - e in alcuni casi solamente alcune particola$r i$ tecniche matematiche - rifiutandosi di tener conto di influenze importanti che tali tecniche non riescono a cogliere.

Michio Morishima, eminente economista matematico, ha anch'egli sostenuto - fa notare esplicitamente Sen - che l'economia è stata molto impoverita dalla cecità di tanti economisti davanti al fatto che molte variabili sfuggono alle strutture matematiche già esistenti. Altri studiosi hanno sostenuto, con una certa plausibilità - continua Sen che gli economisti matematici scelgono solamente problemi che si prestano al formalismo matematico e che lo fanno innanzitutto per divertirsi o per far colpo sui loro colleghi.

2 Festival della matematica, Roma, 13 marzo 2008. 
In sintesi - conclude Sen - l'economia e le scienze sociali hanno per molto tempo tratto benefici dal ragionamento matematico. Dovrebbe tuttavia essere possibile usarlo senza subirne i limiti.

E' proprio questa la prospettiva alla quale io penso che gli economisti "illuminati" dovrebbero tendere.

\section{BIBLIOGRAFIA}

de Vivo, G. (2004), Da Ricardo e Marx a Produzione di Merci a mezzo di Merci, in Atti dei Convegni Lincei, 200, (Convegno internazionale Piero Sraffa, Roma 11-12 febbraio 2003), pp. 215-234, Roma, Accademia Nazionale dei Lincei.

Domenidò, V. (1962), Una teoria economica neoricardiana, in Giornale degli Economisti e Annali di Economia, Novembre-Dicembre, pp.710-731.

Hicks, J.R. (1976), Revolutions in Economics, in Spiro J. Latsis, Method and Appraisal in Economics, Cambridge, Cambridge University Press.

Keynes, J.M. (1930), Economic Possibilities for Our Grandchildren, in Essays in Persuasion, ripubblicato in: John Maynard Keynes (1972), The Collected Writings of John Maynard Keynes, vol. IX, London, Macmillan.

Keynes, J.M. (1933), Alfred Marshall, in Essays in Biography, ripubblicato in: John Maynard Keynes (1972), The Collected Writings of John Maynard Keynes, vol. X, London, Macmillan.

Keynes, J.M. (1938), Letter to Roy Harrod, in: John Maynard Keynes (1972), The Collected Writings of John Maynard Keynes, vol. XIV, The General Theory and after, Part II, Defence and Development, London, Macmillan.

Kuhn, Thomas (1962), The Structure of Scientific Revolutions, $2^{\text {nd }}$ edition, Chicago, University of Chicago Press.

Manara, C.F., (1968), Il modello di Sraffa per la produzione congiunta di merci a mezzo di merci, in L'industria, n. 1, pp. 3-18, ripubblicato in: Pasinetti (1977).

Newman, P. (1962), Production of Commodities by Means of Commodities, in Schweizerische Zeitschrift fur Volkswirtshaft und Statistik, vol. XCVIII, pp. 58-75.

Pasinetti, L.L. (1973), The Notion of Vertical Integration in Economic Analysis, in: Metroeconomica, vol. 25, pp. 1-29; trad. italiana: La nozione di settore verticalmente integrato nell'analisi economica, in: L.L. Pasinetti (1977), pp. 37-74.

Pasinetti, L.L. (1975), Lezioni di Teoria della Produzione (2 ed. ampliata, 1981), Bologna, il Mulino.

Pasinetti, L.L. (1977) (a cura di), Contributi alla teoria della produzione congiunta, Bologna, il Mulino.

Pasinetti, L.L. (1985), Comment a: Edmond Malinvaud, The identification of Scientific Advances in Economics in: T. Hägerstrand (a cura di), The Identification of Progress in Learning, (Atti di un Convegno della European Science Foundation, Colmar, Francia 1983), Cambridge, Cambridge University Press, pp. 183-186. 
Pasinetti, L.L. (1993), Dinamica economica strutturale - Un'indagine teorica sulle conseguenze economiche dell'apprendimento umano, Bologna, il Mulino

Pasinetti, L.L. (2004), Sraffa e la matematica: diffidenza e necessità. Quali sviluppi per il futuro?, in: Atti dei convegni Lincei, 200, (Convegno internazionale Piero Sraffa, Roma 11-12 febbraio 2003), pp. 373-383, Roma, Accademia Nazionale dei Lincei.

Pasinetti, L.L. (2005), Pure theory vs. full economic analysis - Sraffa's riddle (interven-

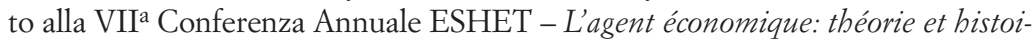
re, Parigi, 30 gennaio- $1^{\circ}$ febbraio 2003), pubblicato come From pure theory to full economic analysis - A place for the economic agent in Cabiers d'Économie Politique - histoire de la pensée et théories, pp. 211-216.

Pasinetti, L.L. e Roncaglia A. (2006), Le scienze umane in Italia: il caso dell'economia politica, in Rivista Italiana degli Economisti, XI, pp. 461-499 (l'articolo contiene la relazione presentata il 27 ottobre 2006 a Roma presso l' Accademia Nazionale dei Lincei in occasione del Convegno Le Scienze Umane in Italia: prospettive per la ricerca e l'alta formazione, organizzato dall'Accademia Nazionale dei Lincei e dall'Istituto di Scienze Umane di Firenze).

Schefold, B. (1971), Piero Sraffas Theorie der Kuppelproduktion des Kapitals und der Rente, dissertazione di $\mathrm{PhD}$, Università di Basilea, parte principale ripubblicata in: Mr. Sraffa on Joint Production, Londra, Unwin Hyman, 1989.

Sen, Amartya K., L'economia sedotta dai numeri, intervento al Festival della matematica, Roma, 13 marzo 2008, riportato dal supplemento culturale de Il Sole 24 Ore domenica 9 marzo 2008.

Sraffa, P. (1960), Production of Commodities by means of Commodities - Prelude to a critique of economic theory, Cambridge, Cambridge University Press. Versione italiana: Produzione di Merci a mezzo di Merci - Premesse a una critica della teoria economica, Torino, Einaudi. 